\title{
Reliable Fault Classification of Induction Motors Using Texture Feature Extraction and a Multiclass Support Vector Machine
}

\author{
Jia Uddin, ${ }^{1}$ Myeongsu Kang, ${ }^{1}$ Dinh V. Nguyen, ${ }^{2}$ and Jong-Myon Kim ${ }^{1}$ \\ ${ }^{1}$ School of Electrical Engineering, University of Ulsan, Building No. 7, Room No. 308, 93 Daehak-ro, Nam-gu, \\ Ulsan 680-749, Republic of Korea \\ ${ }^{2}$ School of Computer Engineering, Hanoi University of Science and Technology, Hanoi 010000, Vietnam
}

Correspondence should be addressed to Jong-Myon Kim; jongmyon.kim@gmail.com

Received 18 May 2014; Accepted 15 June 2014; Published 29 June 2014

Academic Editor: Jong-Hyuk Park

Copyright (C) 2014 Jia Uddin et al. This is an open access article distributed under the Creative Commons Attribution License, which permits unrestricted use, distribution, and reproduction in any medium, provided the original work is properly cited.

\begin{abstract}
This paper proposes a method for the reliable fault detection and classification of induction motors using two-dimensional (2D) texture features and a multiclass support vector machine (MCSVM). The proposed model first converts time-domain vibration signals to $2 \mathrm{D}$ gray images, resulting in texture patterns (or repetitive patterns), and extracts these texture features by generating the dominant neighborhood structure (DNS) map. The principal component analysis (PCA) is then used for the purpose of dimensionality reduction of the high-dimensional feature vector including the extracted texture features due to the fact that the high-dimensional feature vector can degrade classification performance, and this paper configures an effective feature vector including discriminative fault features for diagnosis. Finally, the proposed approach utilizes the one-against-all (OAA) multiclass support vector machines (MCSVMs) to identify induction motor failures. In this study, the Gaussian radial basis function kernel cooperates with OAA MCSVMs to deal with nonlinear fault features. Experimental results demonstrate that the proposed approach outperforms three state-of-the-art fault diagnosis algorithms in terms of fault classification accuracy, yielding an average classification accuracy of $100 \%$ even in noisy environments.
\end{abstract}

\section{Introduction}

Induction motors are widely used in rotary machinery systems, including both heavy-and light-duty machinery [1], and play an important role in the industry due to their hardiness, low cost, and low maintenance requirements. An induction motor usually falls out of service due to the following reasons: the application of an unexpected heavy load, unsuitable or inadequate lubrication, and ineffective sealing. With the increase in the production capabilities of current manufacturing systems, machines are expected to run continuously, making unexpected interruptions due to machine failure being more costly than ever. Fault diagnosis for industrial induction motors is therefore a significant issue. Reliable, fast, automated, and state-monitoring schemes have been widely used to identify specific failures in various induction motor components.

In general, feature extraction can be performed by matching similarities between different signals $[2,3]$. In order to extract the features of numerous faults, current, voltage, vibration, infrared thermography, and acoustic emission signals were utilized as sources to monitor fault states [4]. Among the signals, vibration analysis has been the most frequently employed methodology for identifying induction motor faults due to its ability to represent intrinsic information of them [5].

These vibration signals are analyzed in time domain, frequency domain, and time-frequency domain $[6,7]$. The time-domain analysis determines numerous characteristics from a signal waveform, such as the root mean square (RMS), skewness, kurtosis, and crest factor, and applies these parameters in the diagnosis of inductions motors [8]. Although the scalar descriptors extracted from time signals are robust in terms of fluctuating load, they are not consistently effective, particularly in the presence of increased defects [9]. In contrast, frequency analysis considers only the frequency components, neglecting amplitude, where the frequency patterns are closed to the rotation properties of 
the machine. A number of time-frequency techniques have been proposed as a trade-off between the time and frequency domain techniques.

In the traditional approaches, signals are processed as a one-dimensional (1D) representation, where the relationship information between time and frequency coefficients may be easily lost. In addition, the features collected from either the raw or processed signals have large dimensions that usually increase the computational burden of the subsequent classifier and degrade the generalization capability of a classifier. A significant number of well-known classifiers have been used for fault diagnosis, including artificial neural network (ANN), support vector machine (SVM), simplified fuzzy art map (SFAM), and fuzzy art map (FAM). Conventional feature extraction techniques that collect feature vectors from raw signals are not functional in practical scenarios, because load and rotational speed can vary at any instant. Therefore, an efficient approach for feature extraction with an effective dimension reduction technique is essential.

In this paper, a multifault detection and classification approach is proposed that first converts vibration signals to two-dimensional (2D) gray images by transforming the amplitude of the signals into the intensity of the pixels in an image. Due to the fact that the converted gray-level images show texture patterns (or repetitive patterns), the dominant neighborhood structure (DNS) map is generated to extract the texture features of the converted images in this paper. In general, a feature vector including the extracted texture features is high-dimensional and its high dimensionality can degrade classification performance. Hence, this paper exploits a principal component analysis (PCA) for dimensionality reduction of the feature vector. Finally, the reduced feature vector with discriminative fault features is used as input for the multiclass support vector machines (MCSVMs) to identify each fault in an induction motor. The proposed approach is compared with three conventional techniques in terms of classification accuracy in both noiseless and noisy environments.

The rest of the paper is organized as follows. Section 2 introduces related works, and Section 3 presents the proposed fault diagnosis method. Section 4 describes a detailed implementation of the proposed approach. Section 5 analyzes the experimental results of the proposed approach and compares the classification accuracy of the proposed approach with other conventional techniques. Finally, Section 6 concludes this paper.

\section{Related Works}

Several fault-state monitoring techniques have been proposed to improve robustness, reliability, and production capacity and reduce maintenance costs $[4,8,10-19]$. The proposed techniques include short-time Fourier transform (STFT), scale-invariant feature transform (SIFT), wavelet packet transform (WPT), Wigner-Ville distribution (WVD), Gabor filter, Hilbert Huang transform (HHT), empirical mode decomposition (EMD), local mean decomposition (LMD), and local characteristic-scale decomposition (LCD).

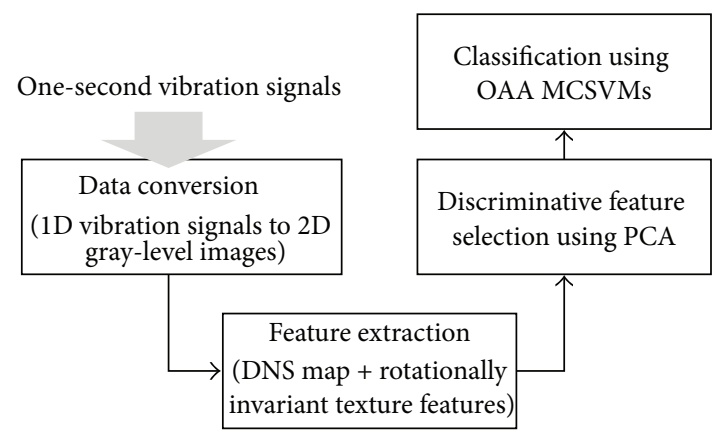

FIGURE 1: A block diagram of the proposed fault diagnosis methodology.

Lei et al. proposed an intelligent classification method using WPT and EMD [10]. Dimensionless time-domain feature vectors were extracted from each of the original vibration signals and preprocessed to form a combined feature set. In [11], a systematic procedure using an adaptive learning system such as ANNs [20] was proposed for fault diagnosis in induction motors, where the time-domain features were calculated from the input signals. William and Hoffman proposed an early fault detection approach using the zerocrossing time-domain features [12]. These features were collected from the input during the zero-crossing intervals. As a result, estimation of the rotational frequency was not required. Rodriguez-Donate et al. proposed a quantitative general methodology for online monitoring of an induction motor and an automatic identification of multiple faults based on the startup vibration transient analysis [4]. Yang and Kim proposed a fault diagnosis model using the Dempster-Shafer theory, a combinational rule using current and vibration signals concurrently to extract feature vectors [13]. Bafroui and Ohadi proposed a fault diagnosis model to process nonstationary vibration signals, where a resample technique at a constant angle increment is combined with the continuous wavelet transform (CWT) and measured the numerous statistical parameters from the wavelet coefficients [19]. An improved HHT-based detection methodology was proposed in [21] for removing undesirable intrinsic mode functions (IMFs), where noisy IMFs were selected based on a threshold value. A new self-adaptive time-frequency domain method, LMD, was proposed that produces a number of product functions, and a multiscale entropy of each product function was utilized to calculate the feature vectors [22]. Zheng et al. proposed a new nonstationary fault diagnosis method, LCD, which decomposes the vibration signal adaptively in a series of intrinsic scale components (ISC) for different scales [23].

\section{Proposed Model}

Figure 1 presents a block diagram of the proposed model consisting of the following four blocks: data conversion, texture feature extraction, texture feature reduction, and classification.

First, data conversion process is carried out in order to produce $2 \mathrm{D}$ gray-level images from $1 \mathrm{D}$ vibration signals. 


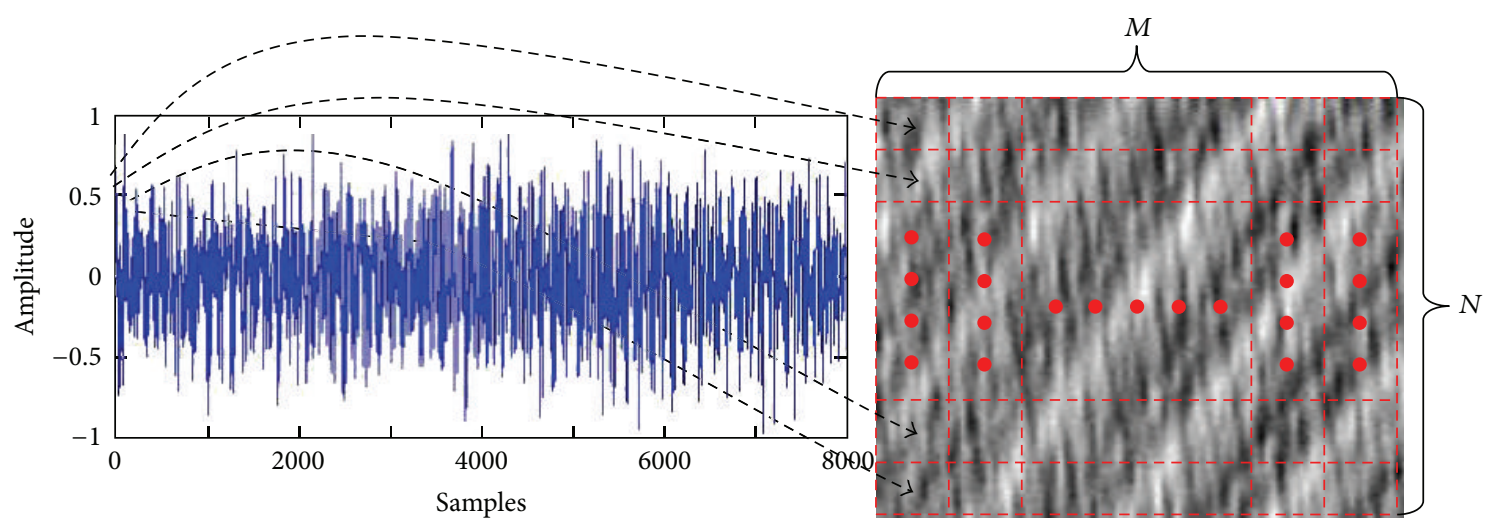

FIGURE 2: Data conversion process to represent a two-dimensional gray-level image from a one-dimensional vibration signal.

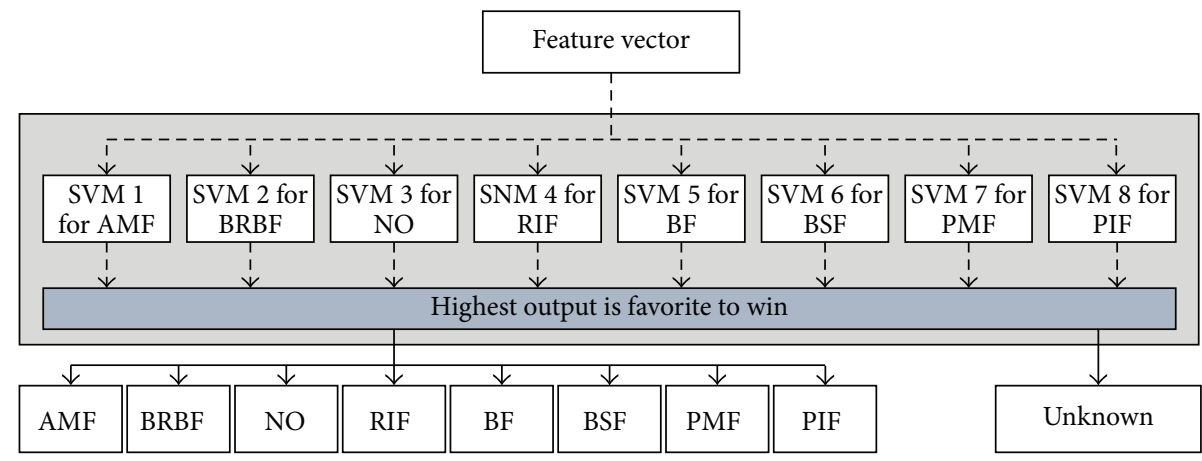

FIGURE 3: OAA MCSVMs for identifying induction motor failures.

In the data conversion process, the amplitude of each sample of the vibration signal is normalized to range from 0 to 255 , and the normalized amplitude of each sample becomes the intensity of the corresponding pixel, as shown in Figure 2. The coordinate of the corresponding pixel for the $i$ th sample of the vibration signal is pixel $(j, k)$, where $j=\operatorname{modulo}(i / M), k=$ floor $(i / M)+1, M$ is the column length of an $M \times N$ image, and $N$ is the row length of an $M \times N$ image.

We then generate a DNS map to extract texture features in the converted image [24] and the overall process of the DNS map generation can be summarized as follows.

Step 1. The center pixel is located at the center of the search window and the other pixels in the search window are neighboring pixels. To generate a DNS map, vectors $v\left(N_{i}\right)$ and $v\left(N_{j}\right)$ are first created, where $v\left(N_{i}\right)$ is the vector of the neighborhood pixel values around the pixel $i$ surrounded by the $n \times n$ neighborhood window, $v\left(N_{j}\right)$ is the vector of neighboring pixels within the neighborhood window, the pixel $i$ refers to the pixel intensity at the center of the $m \times m$ search window, and the pixel $j$ indicates each pixel value of the search window.

Step 2. Euclidean distances are then computed between the two generated vectors and all of the pixels in the search window are replaced with Euclidean distances. In this study, the center pixel is also considered to be a neighborhood pixel and the Euclidean distance can be 0 if $v\left(N_{i}\right)=v\left(N_{j}\right)$.
Step 3. The spacing interval is the distance between pixels used to build the DNS map, which affects the resolution at which the global neighborhood similarity is captured. To produce the DNS map at a given scale of resolution, it is important to have a sufficient number of neighborhood structure maps. This can be achieved by moving the predefined $m \times m$ search window at a certain spacing interval between the previous search window and the current search window. The spacing interval was experimentally determined in this study, which is set to 5 .

Since the converted texture image can be rotated by a certain rotation angle, it is necessary to extract rotationally invariant features. To deal with this problem, the DNS map values are sampled on circumferences of concentric circles of various radii centered at the center pixel of the map. However, the dimensionality of a feature vector including these texture features is high, which can degrade classification performance. Thus, selection of the most discriminative feature is vital for reliable fault diagnosis. For the purpose of dimensionality reduction in this study, we utilize PCA [25] to select the most distinctive features between classes. The PCA algorithm reduces the dimension of the feature vectors by selecting only the principal components, where a principal component is defined by a linear transformation of the original variables into a new set of uncorrelated variables. PCA finds a new set of feature vectors with principal directions (PDs) and insignificant directions (IDs), where PDs have 
Table 1: Description of induction motor faults.

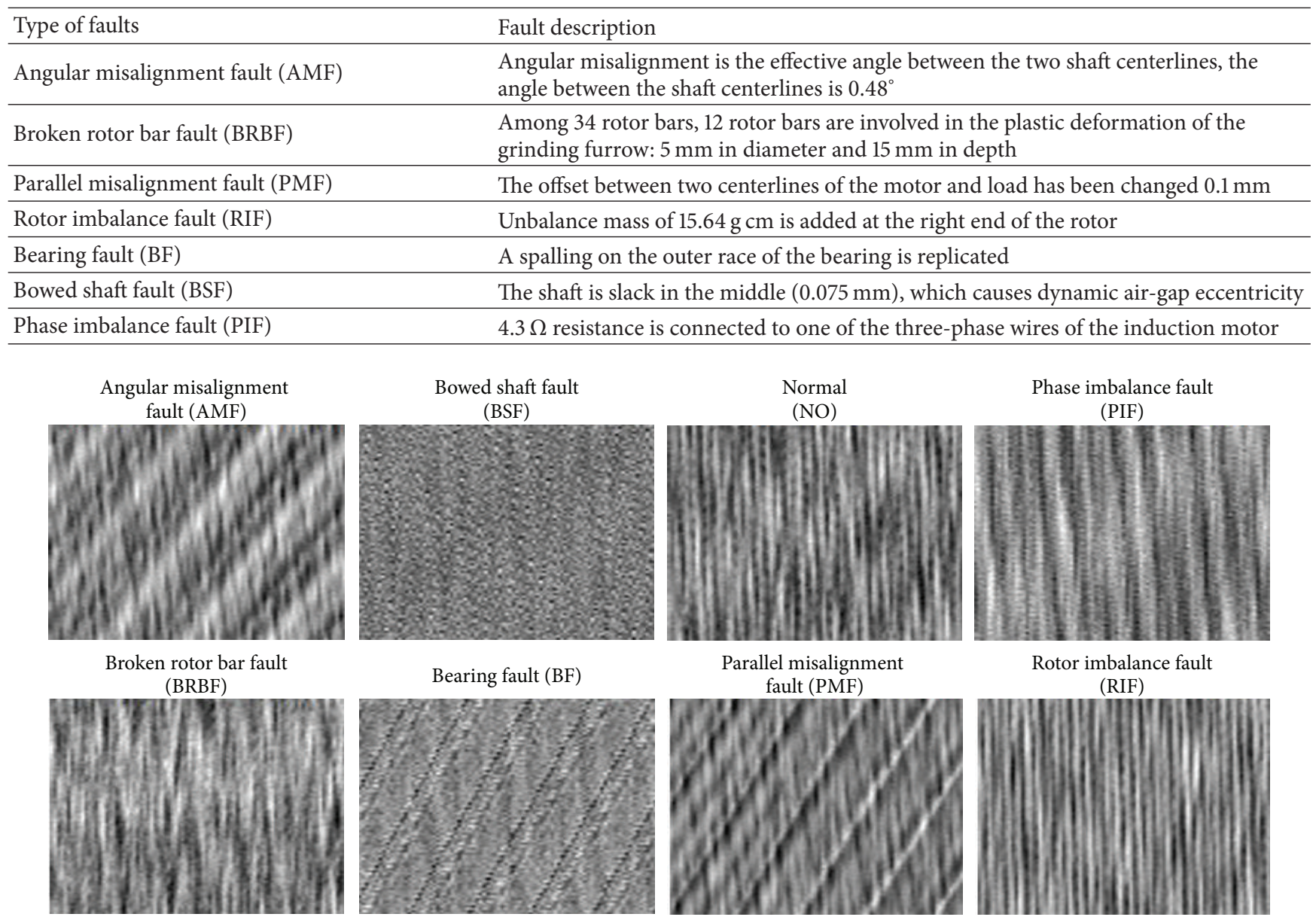

FIGURE 4: Examples of 2D representation of vibration signals.

large covariance and IDs have small covariance. Among these new feature vectors, only the most significant features are utilized as inputs to the classifier.

Finally, we utilize multiclass SVM (MCSVM) as a classifier, where the Gaussian radial basis function is used as a function kernel to ensure efficient nonlinear classification between faults. In order to design MCSVMs, the following three approaches can be considered: one-againstall (OAA), one-against-one (OAO), and one-acyclic-graph (OAG). Among them, the OAA method is employed, which is one of the most popular and simplest techniques for multiclass classifiers. In the OAA approach, each SVM structure discriminates one class from the others, and the final decision can be made by selecting the SVM structure yielding the highest output value.

In order to design OAA MCSVMs, each SVM structure is separately evaluated to achieve the maximum classification accuracy for its own class. Then, all SVM structures cooperate together to make a final decision. If none of the classes can recognize what type of fault the input signal is, the final decision is assigned to unknown. OAA MCSVMs for classifying faults are illustrated in Figure 3. To train and test OAA MCSVMs, it is necessary to build a training dataset
TABLE 2: Parameter setups for generating a DNS map.

\begin{tabular}{lc}
\hline Parameters & Values \\
\hline Searching window size & $21 \times 21$ \\
Neighbor window & $13 \times 13$ \\
Number of central pixels & 144 \\
Size of DNS map & $21 \times 21$ \\
Gap between two central pixels & 5 pixels \\
\hline
\end{tabular}

using $50 \%$ of the 105 vibration signals for each fault, including normal signals and a test dataset with the remaining signals in this study (e.g., 53 one-second vibration signals are used as the training data set, and 52 one-second vibration signals are used as the test data set).

\section{Implementation of the Proposed Model}

In our experiment, we utilized eight types of signals: angular misalignment fault (AMF), bearing fault (BF), bowed shaft fault (BSF), phase imbalance fault (PMF), broken rotor bar fault $(\mathrm{BRBF})$, rotor imbalance fault (RIF), phase imbalance 


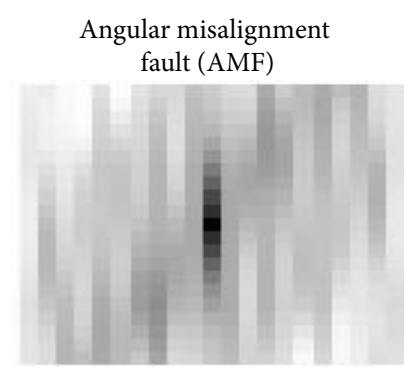

Broken rotor bar fault (BRBF)

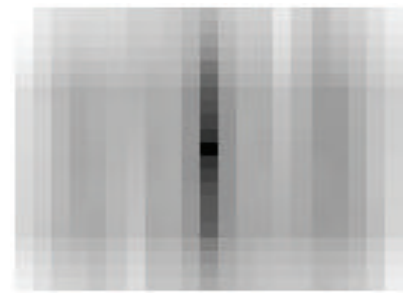

Bowed shaft fault (BSF)

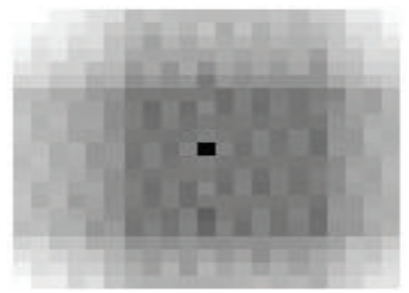

Bearing fault (BF)

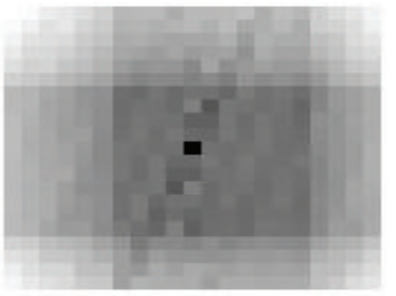

Normal

(NO)

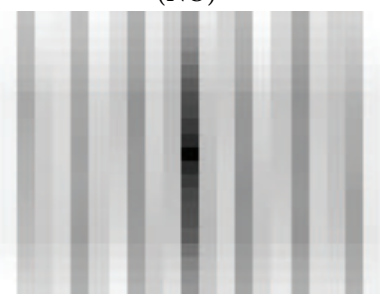

Parallel misalignment fault (PMF)

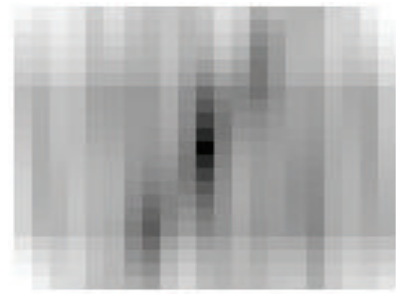

Phase imbalance fault (PIF)

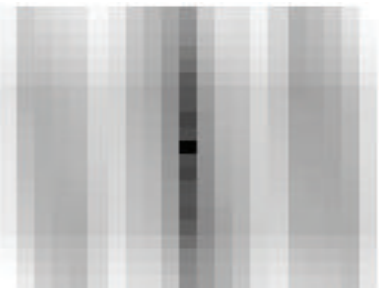

Rotor imbalance fault (RIF)

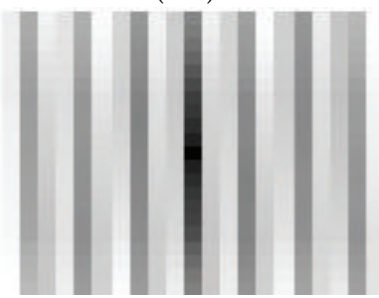

FIGURE 5: DNS maps obtained from the converted texture images.

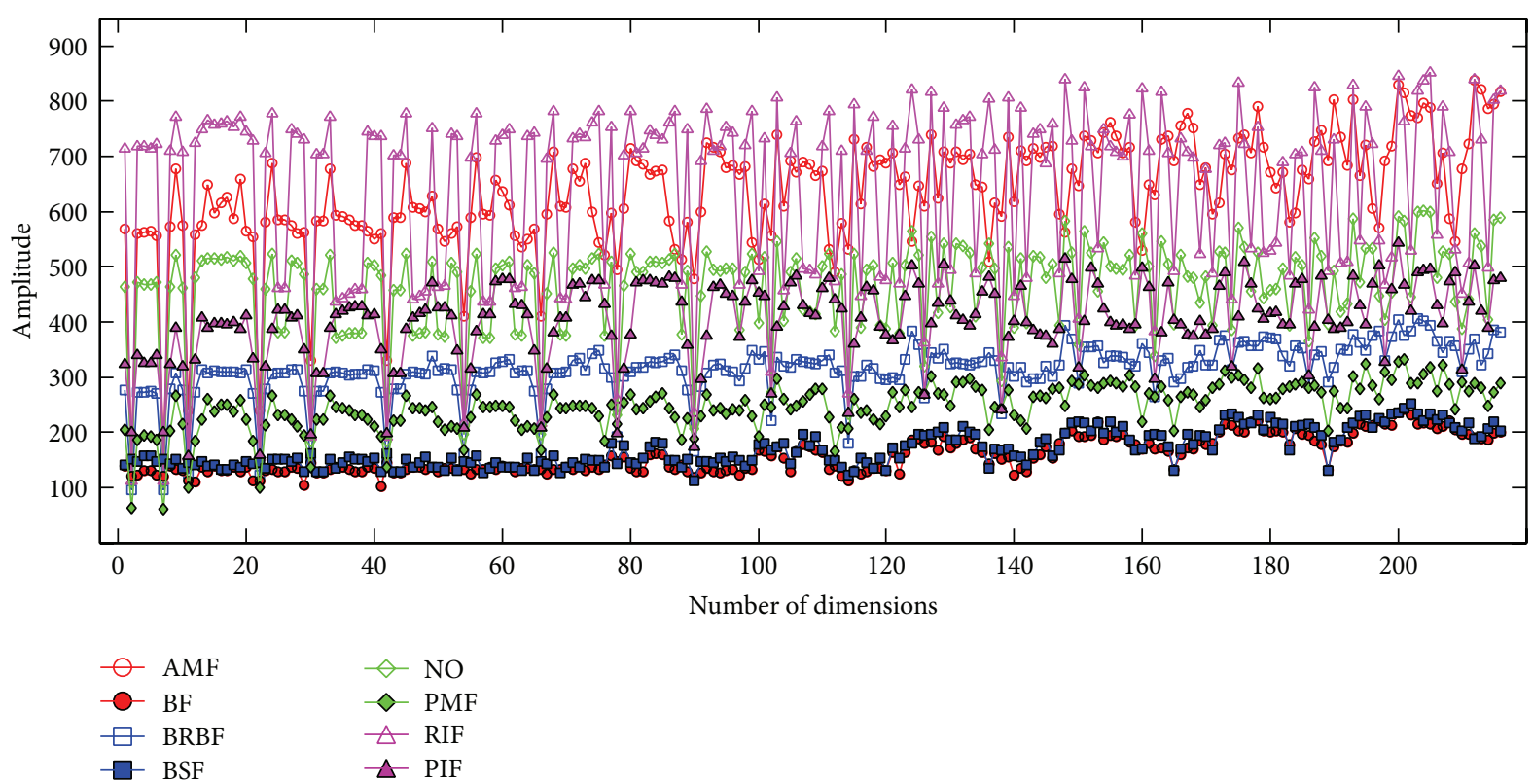

FIGURE 6: $1 \times 216$ Feature vectors including rotationally invariant features for various induction motor faults.

fault (PIF), and healthy (NO). We acquired one-second 105 vibration signals for each fault condition from the accelerometer located at the axial direction of the induction motor and Table 1 presents a brief description of each fault used in this study.

To efficiently observe the relationship of samples, this paper converts one-second $1 \mathrm{D}$ time-domain vibration signal into a 2D gray-level image with a size of $89 \times 89$ and Figure 4 shows a $2 \mathrm{D}$ representation of vibration signals for each fault condition. Likewise, Table 2 shows parameters (e.g., search window size, neighborhood window size, and so on) for generating a DNS map and Figure 5 depicts the DNS map for each fault condition.

As mentioned in previous section, DNS map values are sampled on circumferences of concentric circles of various radii centered at the center pixel of the map. For a DNS map of size $21 \times 21$, ten circles are used in order to cover all of the DNS map regions. For circles of radii greater than two pixels, 24 uniform angular measurements are extracted. Only 8 and 16 measurements are extracted from the first two innermost circles. Therefore, the number of dimensions of the feature vector is $216(=8+16+8 \times 24)$ and Figure 6 illustrates 


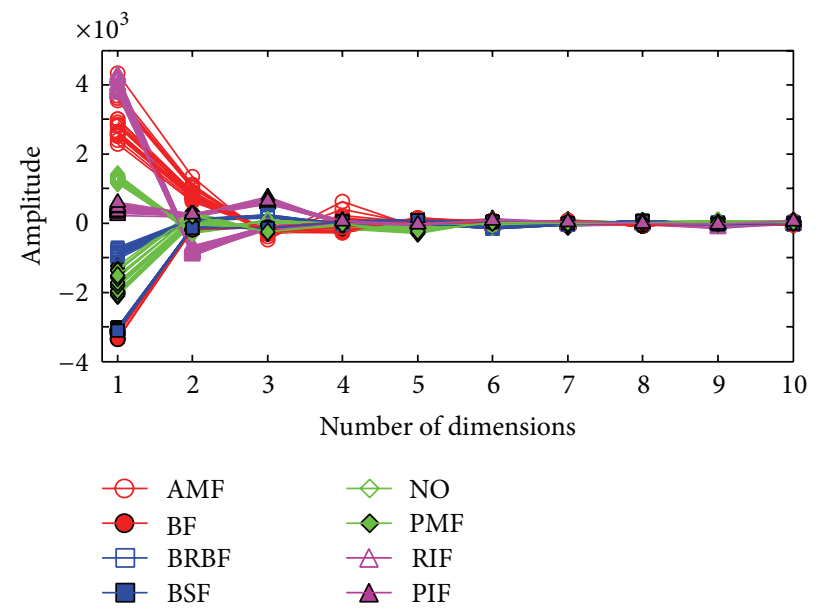

FIgURE 7: Patterns of principal components of each induction motor fault.

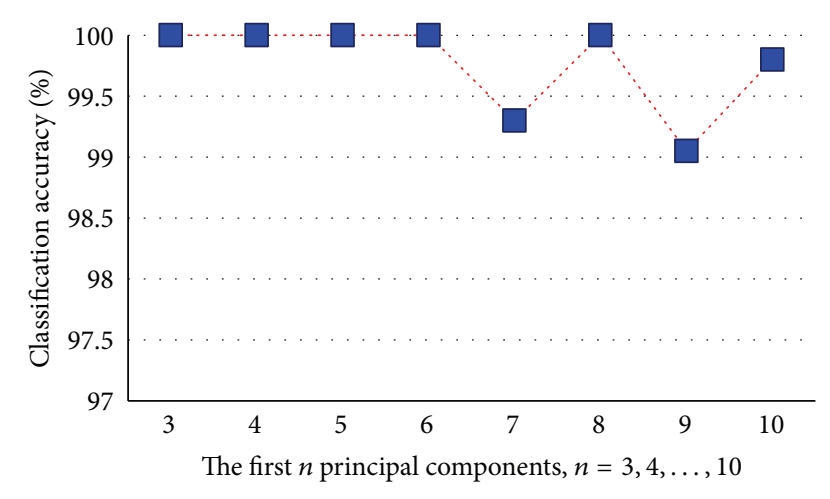

Figure 8: Classification accuracy with different numbers of principal components.

$1 \times 216$ feature vectors including rotationally invariant features for each fault condition.

It was found that computational complexity typically increases and overall classification accuracy decreases with the high-dimensional feature vector. Thus, we employed the PCA to reduce computational complexity and improve the overall classification accuracy by selecting the most discriminative features from the high-dimensional feature vector. Figure 7 demonstrates magnitudes of several principal components after applying the PCA to the high-dimensional feature vectors. For the PCA, it is necessary to determine the most influential component for fault diagnosis and Figure 8 shows the classification results with different numbers of principal components. As depicted in Figure 8, the proposed fault diagnosis methodology achieves maximum classification accuracies when we utilize first three to six principal components as inputs of OAA MCSVMs and consequently this paper utilizes the first three principal components as fault features in this study providing both the highest classification performance and the lowest computational time.

In the observation and testing process, we utilized SVM with a Gaussian radial basis function (RBF) kernel [17] because the RBF kernel shows an exceptional ability to
TABLE 3: Optimal sigma values of each SVM strutcure.

\begin{tabular}{lcc}
\hline Faults & Optimal range & Selected values \\
\hline AMF (SVM1: class 1) & $0.2<\sigma<2.0$ & 1.0 \\
BRBF (SVM2: class 2) & $0.1<\sigma<2.0$ & 0.9 \\
NO (SVM3: class 3) & $0.1<\sigma<1.4$ & 0.6 \\
RIF (SVM4: class 4) & $0.1<\sigma<1.9$ & 1.0 \\
BF (SVM5: class 5) & $0.1<\sigma<0.3$ & 0.2 \\
BSF (SVM6: class 6) & $0.1<\sigma<0.4$ & 0.2 \\
PMF (SVM7: class 7) & $0.1<\sigma<1.2$ & 0.6 \\
PIF (SVM8: class 8) & $0.1<\sigma<1.1$ & 0.5 \\
\hline
\end{tabular}

handle the nonlinear problem inherent in the time-varying vibration signals caused by motor slippage and variable rotational speed. The Gaussian radial basis function kernel is represented as follows [26]:

$$
k\left(s v_{i}, s v_{j}\right)=\exp \left(-\frac{\left\|s v_{i}-s v_{j}\right\|^{2}}{2 \sigma^{2}}\right),
$$

where $k\left(s v_{i}, s v_{j}\right)$ is the RBF kernel, $s v_{i}$ and $s v_{j}$ are the input feature vectors, and $\sigma$ is a parameter set by users to determine the effective width of the RBF kernel. The selection of an optimal $\sigma$ value of the RBF kernel is an important issue. Therefore, this paper explores the impact of $\sigma$ values on the classification performance in the range from 0.1 to 2 at intervals of 0.1 . Table 3 presents the optimal range of $\sigma$ values for each SVM in order to classify faults of the induction motor.

\section{Experimental Results}

The performance of the proposed model is evaluated in terms of true positive (TP) and false positive (FP) classification accuracy [27], where TP represents the number of faults in class $i$ that are correctly classified into class $i$, and FP is the number of faults in other classes that are incorrectly classified into class $i$. Furthermore, since the vibration signals 
TABLE 4: True positive and false positive of classification accuracy without noise.

\begin{tabular}{|c|c|c|c|c|c|c|c|c|c|}
\hline \multirow{2}{*}{ Algorithm } & \multicolumn{8}{|c|}{ Average classification accuracy in terms of true positive and false positive } & \multirow{2}{*}{ Average } \\
\hline & AMF & $\mathrm{BRBF}$ & NO & RIF & $\mathrm{BF}$ & BSF & PMF & PIF & \\
\hline \multicolumn{10}{|c|}{ Algorithm 1 [11] } \\
\hline $\mathrm{TP}$ & 91.35 & 91.54 & 84.23 & 90.38 & 96.73 & 98.46 & 97.11 & 80.19 & 91.24875 \\
\hline FP & 1.54 & 1.24 & 2.17 & 0.80 & 0.22 & 0.47 & 0.63 & 3.24 & 1.28875 \\
\hline \multicolumn{10}{|c|}{ Algorithm 2 [10] } \\
\hline $\mathrm{TP}$ & 98.85 & 93.85 & 90.00 & 100.00 & 100.00 & 100.00 & 96.73 & 99.04 & 97.30875 \\
\hline $\mathrm{FP}$ & 0.00 & 1.59 & 1.02 & 0.00 & 0.00 & 0.00 & 0.76 & 0.00 & 0.42125 \\
\hline \multicolumn{10}{|c|}{ Algorithm 3 [4] } \\
\hline $\mathrm{TP}$ & 100.0 & 100.0 & 100.0 & 100.00 & 100.00 & 100.00 & 100.00 & 100.0 & 100 \\
\hline $\mathrm{FP}$ & 0.00 & 0.00 & 0.00 & 0.00 & 0.00 & 0.00 & 0.00 & 0.00 & 0 \\
\hline \multicolumn{10}{|c|}{ Proposed approach } \\
\hline $\mathrm{TP}$ & 100.0 & 100.00 & 100.00 & 100.00 & 100.00 & 100.00 & 100.00 & 100.0 & 100.00 \\
\hline FP & 0.00 & 0.00 & 0.00 & 0.00 & 0.00 & 0.00 & 0.00 & 0.00 & 0.00 \\
\hline
\end{tabular}

TABLE 5: True positive and false positive of classification accuracy with noise (SNR $=15 \mathrm{~dB}$ ).

\begin{tabular}{|c|c|c|c|c|c|c|c|c|c|}
\hline \multirow{2}{*}{ Algorithm } & \multicolumn{8}{|c|}{ Average classification accuracy in terms of true positive and false positive } & \multirow{2}{*}{ Average } \\
\hline & AMF & $\mathrm{BRBF}$ & NO & RIF & $\mathrm{BF}$ & BSF & PMF & PIF & \\
\hline \multicolumn{10}{|c|}{ Algorithm 1 [11] } \\
\hline $\mathrm{TP}$ & 91.45 & 88.00 & 84.00 & 90.91 & 96.00 & 98.91 & 95.19 & 79.82 & 90.535 \\
\hline FP & 1.95 & 0.49 & 2.57 & 0.99 & 0.16 & 0.57 & 0.25 & 3.40 & 1.2975 \\
\hline \multicolumn{10}{|c|}{ Algorithm 2 [10] } \\
\hline $\mathrm{TP}$ & 96.15 & 85.57 & 48.26 & 78.46 & 100.00 & 100.00 & 96.92 & 100.0 & 88.17 \\
\hline $\mathrm{FP}$ & 0.00 & 10.83 & 0.73 & 0.03 & 0.00 & 0.000 & 3.18 & 3.65 & 2.3025 \\
\hline \multicolumn{10}{|c|}{ Algorithm 3 [4] } \\
\hline $\mathrm{TP}$ & 34.42 & 0.00 & 7.50 & 100.00 & 100.00 & 100.00 & 91.73 & 91.92 & 65.69625 \\
\hline FP & 0.00 & 0.00 & 7.45 & 23.74 & 2.31 & 4.53 & 0.00 & 0.00 & 4.75375 \\
\hline \multicolumn{10}{|c|}{ Proposed approach } \\
\hline $\mathrm{TP}$ & 100.0 & 94.61 & 100.0 & 100.00 & 100.00 & 100.00 & 100.00 & 100.0 & 100.00 \\
\hline $\mathrm{FP}$ & 0.00 & 0.00 & 0.00 & 0.00 & 0.00 & 0.00 & 0.00 & 0.00 & 0.00 \\
\hline
\end{tabular}

can be influenced by various noise levels in real industrial environment, we evaluate the robustness of the proposed fault diagnosis approach in noisy environments by artificially adding white Gaussian noise to the acquired vibration signals and setting the signal-to-noise ratio (SNR) between the originally acquired vibration signals and the noise-inserted vibration signals at $15 \mathrm{~dB}$ and $20 \mathrm{~dB}$, respectively. To guarantee the reliability of the proposed fault diagnosis approach in terms of classification accuracy, we evaluate it ten times with randomly generated training data and test data at each iteration. The final classification accuracy can be obtained by averaging the total classification accuracies. Tables 4,5 , and 6 show the average classification accuracies of the proposed and conventional algorithms in noiseless and noisy environments.

Experimental results show that the proposed approach achieves $100 \%$ classification accuracy even in noisy environments. We observe that the number of significant pixels does not show abrupt change even if noise is included in the vibration signals to a limited value. In contrast, Algorithm 1
[11], which utilizes the time-domain statistical features (RMS, variance, skewness, and kurtosis) from the vibration signal, provides relatively lower classification accuracy for BRBF, NO, RIF, and PIF than those for AMF, BF, BSF, and PMF in noisy environments. This is due to the characteristics of $\mathrm{BRBF}$, NO, RIF, and PIF which are not distinctive enough each other. Algorithm 2 [10], utilizing a combination of wavelet packet transform (WPT) and empirical decomposition, also fails to provide high classification accuracy in noisy environments due to its limitation in selecting the correct number of significant IMFs from the EMD algorithm. Algorithm 3 [4] uses the reconstruction of discrete wavelet packet nodes with information entropy and also exhibits low classification accuracies in noisy environments due to the nonadaptive selection of the decomposition signal level. Overall, the proposed fault diagnosis model outperforms other conventional algorithms in classification accuracy both with and without a noisy environment. 
TABLE 6: True positive and false positive of classification accuracy with noise (SNR $=20 \mathrm{~dB}$ ).

\begin{tabular}{|c|c|c|c|c|c|c|c|c|c|}
\hline \multirow{2}{*}{ Algorithm } & \multicolumn{8}{|c|}{ Average classification accuracy in terms of true positive and false positive } & \multirow{2}{*}{ Average } \\
\hline & AMF & BRBF & NO & RIF & $\mathrm{BF}$ & BSF & PMF & PIF & \\
\hline \multicolumn{10}{|c|}{ Algorithm 1 [11] } \\
\hline $\mathrm{TP}$ & 91.54 & 88.08 & 84.04 & 90.96 & 95.96 & 99.04 & 95.96 & 79.81 & 90.67375 \\
\hline FP & 1.92 & 0.49 & 2.58 & 0.99 & 0.14 & 0.58 & 0.41 & 3.38 & 1.31125 \\
\hline \multicolumn{10}{|c|}{ Algorithm 2 [10] } \\
\hline $\mathrm{TP}$ & 98.27 & 86.92 & 72.31 & 99.23 & 99.42 & 100.00 & 93.84 & 100.0 & 93.74875 \\
\hline $\mathrm{FP}$ & 0.00 & 3.65 & 1.32 & 0.14 & 0.00 & 0.14 & 0.91 & 1.02 & 0.8975 \\
\hline \multicolumn{10}{|c|}{ Algorithm 3 [4] } \\
\hline $\mathrm{TP}$ & 97.31 & 87.88 & 84.42 & 100.00 & 100.00 & 100.00 & 100.00 & 100.0 & 96.20125 \\
\hline $\mathrm{FP}$ & 0.00 & 0.00 & 1.43 & 2.61 & 0.30 & 0.00 & 0.00 & 0.00 & 0.5425 \\
\hline \multicolumn{10}{|c|}{ Proposed approach } \\
\hline $\mathrm{TP}$ & 100.0 & 100.0 & 100.0 & 100.00 & 100.00 & 100.00 & 100.00 & 100.0 & 100.00 \\
\hline FP & 0.00 & 0.00 & 0.00 & 0.00 & 0.00 & 0.00 & 0.00 & 0.00 & 0.00 \\
\hline
\end{tabular}

\section{Conclusions}

This paper proposed a robust fault detection and classification approach for induction motors using texture feature extraction and OAA MCSVMs. In the proposed approach, time-domain vibration signals were first converted into gray images to exploit texture features from the converted images of each faulty vibration signal. Feature vectors were then calculated from the DNS maps of the images. The PCA was utilized to select the most discriminative features by eliminating the trivial ones. Finally, using the distinctive features as inputs, OAA MCSVMs identify each fault of the induction motor. Experimental results showed that the proposed approach achieves 100\% classification accuracy even in noisy environments.

\section{Conflict of Interests}

The authors declare that there is no conflict of interests regarding the publication of this paper.

\section{Acknowledgments}

This research was supported by the Basic Science Research Program through the National Research Foundation of Korea (NRF) funded by the Ministry of Education, Science, and Technology (NRF-2012R1A1A2043644), and this work was supported by the National Research Foundation of Korea (NRF) Grant funded by the Korean government (MEST) (no. NRF-2013R1A2A2A05004566).

\section{References}

[1] M. Zhao, X. Jin, Z. Zhang, and B. Li, "Fault diagnosis of rolling element bearings via discriminative subspace learning: visualization and classification," Expert Systems with Applications, vol. 41, no. 7, pp. 3391-3401, 2014.

[2] E. Namsrai, T. Munkhdalai, M. Li, J. Shin, O. Namsrai, and K. H. Ryu, "A feature selection-based ensemble method for arrhythmia classification," Journal of Information Processing Systems, vol. 9, no. 1, pp. 31-40, 2013.

[3] K. Goswami, G. S. Hong, and B. G. Kim, "A novel mesh-based moving object detection technique in video sequence," Journal of Convergence, vol. 4, no. 3, pp. 20-24, 2013.

[4] C. Rodriguez-Donate, R. J. Romero-Troncoso, E. Cabal-Yepez, A. Garcia-Perez, and R. A. Osornio-Rios, "Wavelet-based general methodology for multiple fault detection on induction motors at the startup vibration transient," Journal of Vibration and Control, vol. 17, no. 9, pp. 1299-1309, 2011.

[5] L. Batista, B. Badri, R. Sabourin, and M. Thomas, "A classifier fusion system for bearing fault diagnosis," Expert Systems with Applications, vol. 40, no. 17, pp. 6788-6797, 2013.

[6] S. Bansal, S. Sahoo, R. Tiwari, and D. J. Bordoloi, "Multiclass fault diagnosis in gears using support vector machine algorithms based on frequency domain data," Measurement, vol. 46, no. 9, pp. 3469-3481, 2013.

[7] M. Kang and J. M. Kim, "Singular value decomposition based feature extraction approaches for classifying faults of induction motors," Mechanical Systems and Signal Processing, vol. 41, pp. 348-356, 2013.

[8] S. Silas, K. Ezra, and E. B. Rajsingh, "A novel fault tolerant service selection framework for pervasive computing," Journal of Human-Centric Computing and Information Sciences, vol. 2, no. 5, pp. 1-14, 2012.

[9] M. Kedadouche, M. Thomas, and A. Tahan, "Empirical mode decomposition of acoustic emission for early detection of bearing defects," in Advances in Condition Monitoring of Machinery in Non-Stationary Operations, Lecture Notes in Mechanical Engineering, pp. 367-377, Springer, Berlin, Germany, 2014.

[10] Y. Lei, Z. He, and Y. Zi, "Application of an intelligent classification method to mechanical fault diagnosis," Expert Systems with Applications, vol. 36, no. 6, pp. 9941-9948, 2009.

[11] J. Zarei, "Induction motors bearing fault detection using pattern recognition techniques," Expert Systems with Applications, vol. 39, no. 1, pp. 68-73, 2012.

[12] P. E. William and M. W. Hoffman, "Identification of bearing faults using time domain zero-crossings," Mechanical Systems and Signal Processing, vol. 25, no. 8, pp. 3078-3088, 2011.

[13] B.-S. Yang and K. J. Kim, "Application of Dempster-Shafer theory in fault diagnosis of induction motors using vibration 
and current signals," Mechanical Systems and Signal Processing, vol. 20, no. 2, pp. 403-420, 2006.

[14] Z. T. Li and H. Li, "EMD and envelope spectrum based bearing fault detection," Advanced Materials Research, vol. 459, pp. 233237, 2012.

[15] Z. Xu, J. Xuan, T. Shi, B. Wu, and Y. Hu, "Application of a modified fuzzy ARTMAP with feature-weight learning for the fault diagnosis of bearing," Expert Systems with Applications, vol. 36, no. 6, pp. 9961-9968, 2009.

[16] H. Keskes, A. Braham, and Z. Lachiri, "Broken rotor bar diagnosis in induction machines through stationary wavelet packet transform and multiclass wavelet SVM," Electric Power Systems Research, vol. 97, pp. 151-157, 2013.

[17] S. M. Chang, H. H. Chang, S. H. Yen, and T. K. Shih, "Panoramic human structure maintenance based on invariant features of video frames," Journal of Human-Centric Computing and Information Sciences, vol. 3, article 14, 2013.

[18] S. G. Kim and Y. G. Seo, "A TRUS prostate segmentation using Gabor texture features and snake-like contour," Journal of Information Processing Systems, vol. 9, no. 1, pp. 103-116, 2013.

[19] H. H. Bafroui and A. Ohadi, "Application of wavelet energy and Shannon entropy for feature extraction in gearbox fault detection under varying speed conditions," Neurocomputing, vol. 133, pp. 437-445, 2014.

[20] M. Malkawi and O. Murad, "Artificial neuro fuzzy logic system for detecting human emotions," Journal of Human-Centric Computing and Information Sciences, vol. 3, no. 3, pp. 1-13, 2013.

[21] J. Yan and L. Lu, "Improved Hilber-Huang transform based weak signal detection methodology and its application on incipient fault diagnosis and ECG signal analysis," Journal of Signal Processing, vol. 98, pp. 74-87, 2014.

[22] H. Liu and M. Han, "A fault diagnosis method based on local mean decomposition and multi-scale entropy for rolling bearing," Journal of Mechanism and Machine Theory, vol. 75, pp. 67-78, 2014.

[23] J. Zheng, J. Cheng, and Y. Yang, "A rolling bearing fault diagnosis approach based on LCD and fuzzy entropy," Journal of Mechanism and Machine Theory, vol. 70, pp. 441-453, 2013.

[24] F. M. Khellah, "Texture classification using dominant neighborhood structure," IEEE Transactions on Image Processing, vol. 20, no. 11, pp. 3270-3279, 2011.

[25] H. Abdi and L. J. Williams, "Principal component analysis," Wiley Interdisciplinary Reviews: Computational Statistics, vol. 2, no. 4, pp. 433-459, 2010.

[26] D. Li, I. K. Sethi, N. Dimitrova, and T. McGee, "Classification of general audio data for content-based retrieval," Pattern Recognition Letters, vol. 22, no. 5, pp. 533-544, 2001.

[27] D. Ghimire and J. Lee, "A robust face detection method based on skin color and edges," Journal of Information Processing Systems, vol. 9, no. 1, pp. 141-156, 2013. 


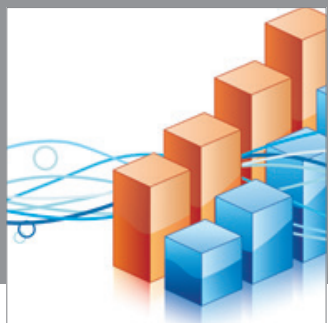

Advances in

Operations Research

mansans

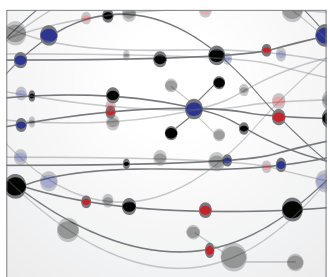

The Scientific World Journal
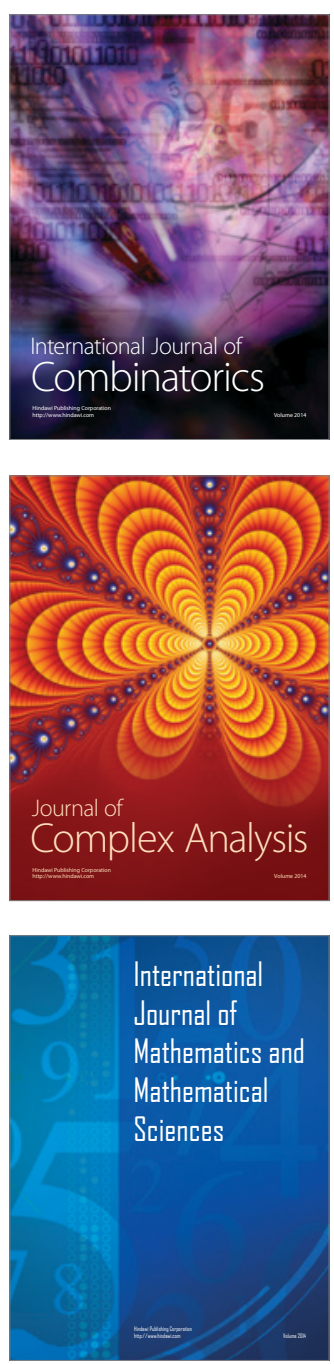
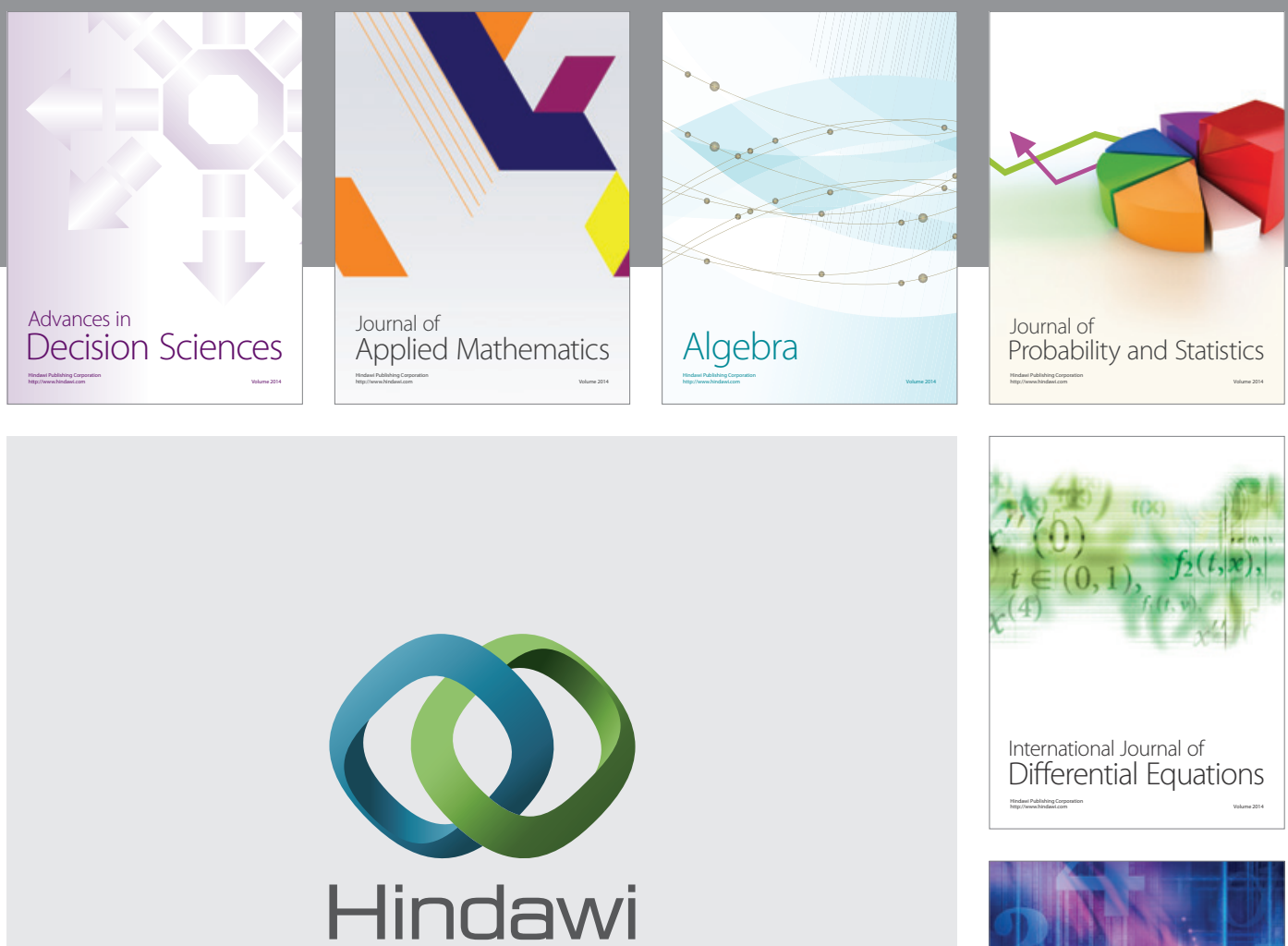

Submit your manuscripts at http://www.hindawi.com
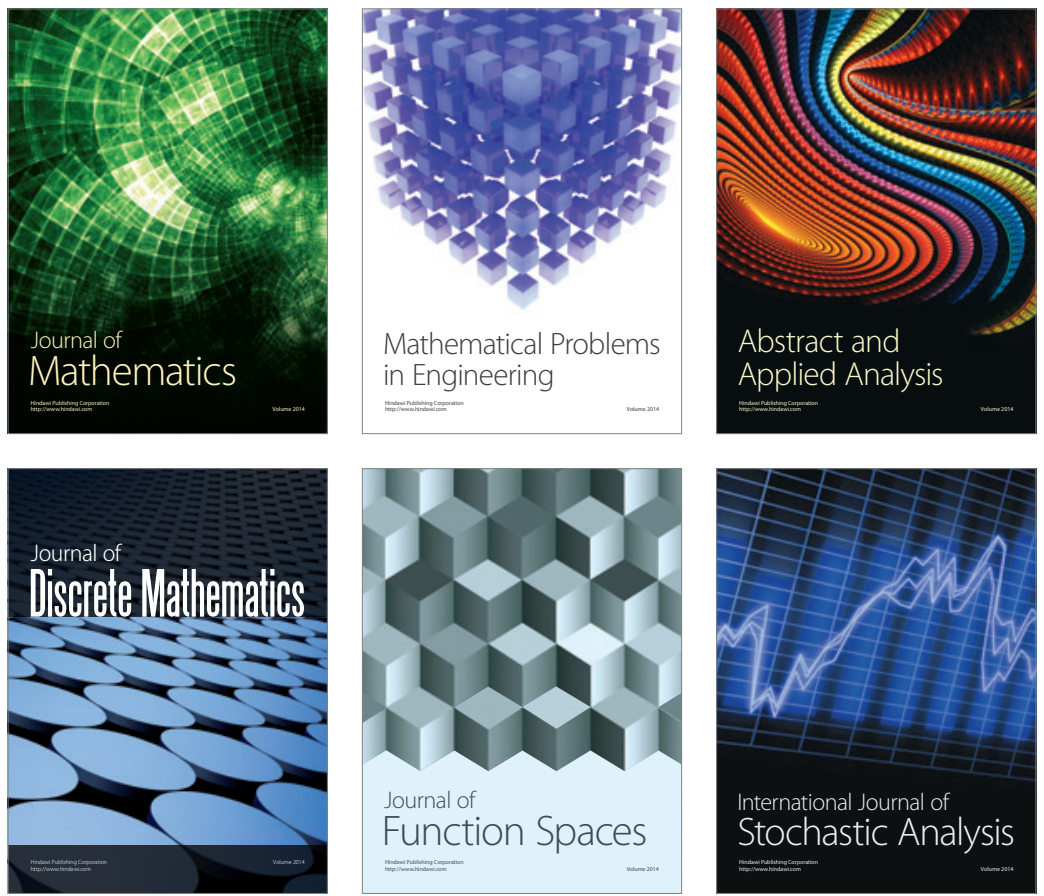

Journal of

Function Spaces

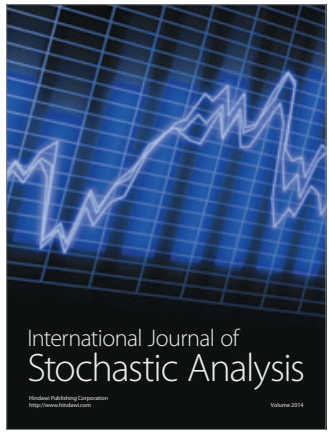

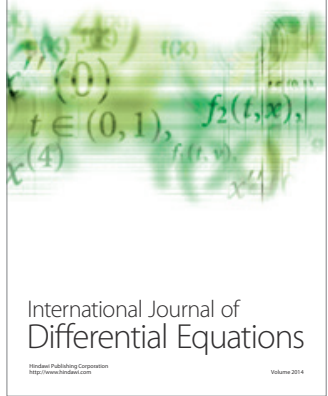
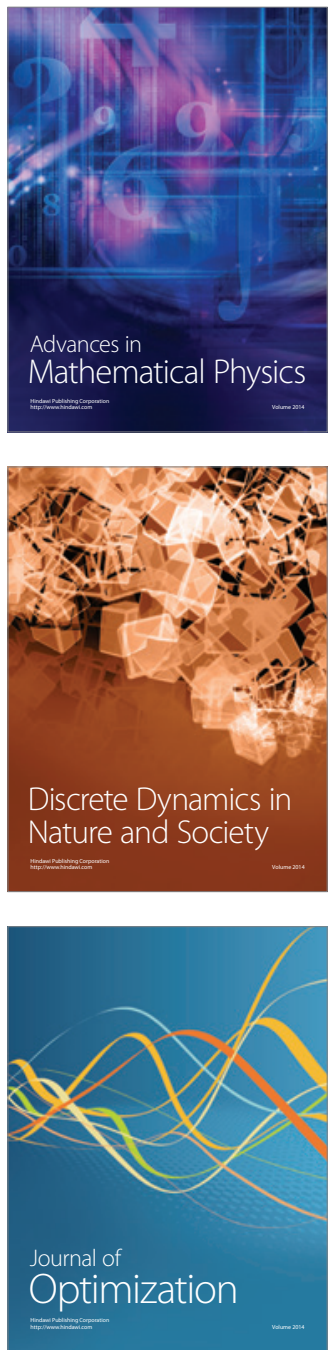